\title{
Quantifying the effect of ionic screening with protein-decorated graphene transistors
}

\author{
Jinglei Ping, ${ }^{\dagger}$ Jin Xi, Jeffery G. Saven, ${ }^{\S}$ Renyu Liu, ${ }^{\ddagger} \&$ A. T. Charlie Johnson ${ }^{*,+}$ \\ ${ }^{\dagger}$ Department of Physics and Astronomy, University of Pennsylvania, Philadelphia 19104, USA \\ ${ }^{\S}$ Department of Chemistry, University of Pennsylvania, Philadelphia, Pennsylvania 19104, USA \\ ${ }^{\ddagger}$ Department of Anesthesiology and Critical Care, University of Pennsylvania, Philadelphia, \\ Pennsylvania 19104, USA \\ *Email: cjohnson@physics.upenn.edu
}

ABSTRACT: Liquid-based applications of biomolecule-decorated field-effect transistors (FETs) range from biosensors to in vivo implants. A critical scientific challenge is to develop a quantitative understanding of the gating effect of charged biomolecules in ionic solution and how this influences the readout of the FETs. To address this issue, we fabricated proteindecorated graphene FETs and measured their electrical properties, specifically the shift in Dirac voltage, in solutions of varying ionic strength. We found excellent quantitative agreement with a model that accounts for both the graphene polarization charge and ionic screening of ions adsorbed on the graphene as well as charged amino acids associated with the immobilized protein. The technique and analysis presented here directly couple the charging status of bound biomolecules to readout of liquid-phase FETs fabricated with graphene or other two-dimensional materials.

Keywords: graphene; redesigned GPCR; ionic screening; field effect transistor 
Introduction

Graphene, a single-atom thick layer of $\mathrm{sp}^{2}$ carbon, is a promising solid-state material for use as an interface to biological systems. Graphene has outstanding biocompatibility ${ }^{1,2}$, and may be fabricated into graphene field effect transistors (GFETs) suitable for decoration with biomolecules such as proteins, or even host active neural cells ${ }^{1}$. In aqueous solution, biomolecules decorating the GFET channel may acquire charges, which will in turn affect the carrier density that governs electron transport through the graphene. When the system is measured in liquid, the electric field associated with charge on the biomolecules is screened by mobile ions and polarization of water molecules; a quantitative model for this effect is essential for understanding the biomolecule/graphene system ${ }^{3,4}$. Prior investigations of this effect were based on either nanowire $\mathrm{FETs}^{3}$, where the channel geometry is not well defined and device yield is relatively low ${ }^{5}$, or single-molecule carbon nanotube $\mathrm{FETs}^{6-8}$ that are not well-suited for scalable fabrication. These elegant studies were mainly performed by measuring real-time fluctuations of the current (or equivalently, resistance or conductance) through the channel due to conformational motion of a single bound protein. The ionic screening effect was understood by the Debye-Hückel model but the effect of protein charging on FET transport properties was treated as an experimentally determined parameter, rather than calculated.

In this paper we demonstrate a protocol to measure the effect of the ionic screening more directly by monitoring changes in the charge carrier density of the GFET channel, instead of changes in the current. We apply the method to bare GFET devices and GFET biosensors that are functionalized with receptor proteins, both measured in solution with ionic strength $c$ of $0.3-$ $150 \mathrm{mM}$. The high end of this range is similar to that of complex biofluids, such as cerebrospinal fluid or blood. The $\mathrm{pH}$ was precisely controlled at 7.0 because GFET conductivity is known to be 
sensitive to this variable ${ }^{9,10}$. Both bare and protein-functionalized GFET devices show the expected ambipolar conduction behavior ${ }^{11}$ in the current-back gate characteristic $\left(I-V_{b g}\right)$, with a conductance minimum at the Dirac voltage, $V_{D}$. Shifts in $V_{D}$ caused by changing ionic strength of the solution or protein functionalization reflect changes in the carrier density of the GFET due to changes in the local electrostatic environment.

In the framework of the electric double layer $(E D L)^{12}$, when bare graphene is in ionic solution, a plane of negative charge ${ }^{9,13}$, the Stern plane, adsorbs and is separated from the graphene by a "Stern layer" with thickness $\sim 0.3-0.4 \mathrm{~nm}^{9,10,14}$, where the molecular density of water is lowered by $97 \%$ compared to the bulk ${ }^{15-17}$. The Stern plane acts as a "chemical gate" that induces a compensating charge density in the graphene and causes $V_{D}$ to shift. The size of the compensating charge in the GFET and associated $V_{D}$ shift depend upon the screening properties of the "diffuse layer" where the electric and osmotic potentials are balanced. The electric potential decays exponentially with distance in the diffuse layer, $={ }_{d} e^{(z) / D}$, where $z$ is the distance from the GFET, $\quad{ }_{d}$ is the potential at the Stern plane, and $\quad{ }_{D}$ is the Debye screening length (See Supporting information for details of the calculation of ${ }_{D}$ ). In the work presented here, the observed shifts of $V_{D}$ for bare GFET devices with respect to ionic strength agree well with theories of the electronic double layer. For protein-functionalized GFETs, we find excellent agreement with a quantitative model for the Dirac voltage shift that includes both ionic screening and the effect of chemical gating ${ }^{18}$ associated with charges on proteins bound to the GFET surface. Thus we confirm that the Dirac voltage shift reflects the electrostatic chemical gating effect rather than Faradaic charge transfer ${ }^{19,20}$. The techniques and analytical methods developed here represent significant progress towards quantitative understanding of nanoenabled sensor systems based on graphene $e^{21,22}$ and may be generalized for liquid-phase 
measurements of protein-functionalized biosensors based on other two-dimensional materials such as $\mathrm{MoS}_{2}$ and $\mathrm{WS}_{2}{ }^{23,24}$.

Material and Methods
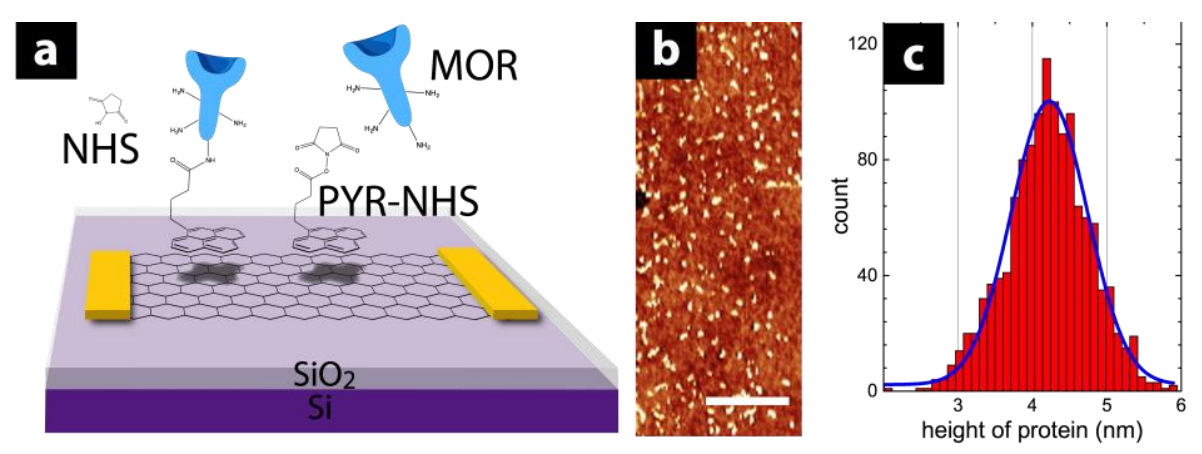

Figure 1. (a) Schematic of chemistry used to immobilize water-soluble mu-opioid receptors (wsMORs) on graphene with 1-Pyrenebutyric acid N-hydroxysuccinimide ester ("PYR-NHS"). (b) Atomic force micrograph shows the high efficiency of the immobilization. The scale bar is 0.5 $\mu \mathrm{m}$. (c) Histogram of measured heights of wsMORs obtained from the AFM images along with a Gaussian fit (blue line).

We use a low-contamination fabrication method ${ }^{22}$ that minimizes contact between the transferred graphene layer and chemicals typically used in device patterning, to avoid negative impact of lithographic processing on electrical and chemical properties of graphene as well as its biological compatibility (See Supporting information for details of Methods). The GFETs have very good transport properties with mobility $\sim 2000 \mathrm{~cm}^{2} \mathrm{~V}^{-1} \mathrm{~s}^{-1}$. We functionalized the GFETs with a computationally redesigned, water-soluble variant of the human mu-opioid receptor (MOR) variant, referred to as WSMOR ${ }^{25,26}$. The redesigned variant of the MOR used in this experiment has a 3D-structure similar to that of the wild-type receptor, and it is readily expressed in $E$. coli 
and purified using standard techniques ${ }^{25}$, without the need for a membrane or membrane surrogate such as a nanodisc ${ }^{27,28}$. Physiologically, the MOR is involved in pain and reward recognition pathways, and it has high affinity for endogenous and exogenous opioids such as heroin, morphine, $\beta$-endorphin, encephalin, and $\beta$-FNA. For these reasons, the wsMORgraphene biosensor is of potential interest for application in human performance monitoring or drug enforcement. The wsMOR has been previously used in a graphene biosensor construct, where its affinity for the drug target naltrexone was confirmed, demonstrating the stability of the wsMOR at ionic strengths as low as $0.3 \mathrm{mM}^{22}$.

Observed changes in graphene transport properties are attributed to the variation of electrostatics caused by wsMOR binding. We use 1-Pyrenebutyric acid N-hydroxysuccinimide ester (PYR-NHS, Sigma-Aldrich) as a bifunctional linker ${ }^{30,31}$ to functionalize GFETs with wsMOR (See Methods in Supporting information). Atomic Force Microscopy shows that the density of wsMOR bound to the GFET surface is $\sim 115 \mu \mathrm{m}^{-2}$ (Fig. 1b). The height histogram of bound wsMOR (Fig. 1c) is well fit by a Gaussian distribution with mean value $\sim 4.2 \mathrm{~nm}$, consistent with the known molecular weight $(\sim 46 \mathrm{kDa})$ and structure of wsMOR.

\section{Results and Discussion}

In the framework of the electrical double layer $(E D L)^{12}$, when bare graphene is in ionic solution, a plane of negative ions ${ }^{9,13}$, the Stern plane, adsorbs such that the nuclei are separated from the graphene by a "Stern layer" with thickness $\sim 0.3-0.4 \mathrm{~nm}^{9,10,14}$, where the molecular density of water is lowered by $97 \%$ compared to the bulk ${ }^{15-17}$. The Stern plane acts as a chemical gate that induces a compensating charge density in the graphene and causes a shift in the Dirac 
voltage, $V_{D}$, the back-gate voltage where the conductance of the graphene is a minimum.

Beyond the Stern layer, there is a diffuse layer of ions where the electric and osmotic potentials are balanced. The electric potential decays exponentially with distance into the solution in the diffuse layer, $={ }_{d} e^{(z) / D}$, where $z$ is the distance from a point in the solution to the graphene, ${ }_{d}$ is the potential at the Stern plane, and ${ }_{D}$ is the Debye screening length (See Supporting information for details of the calculation of ${ }_{D}$ ).

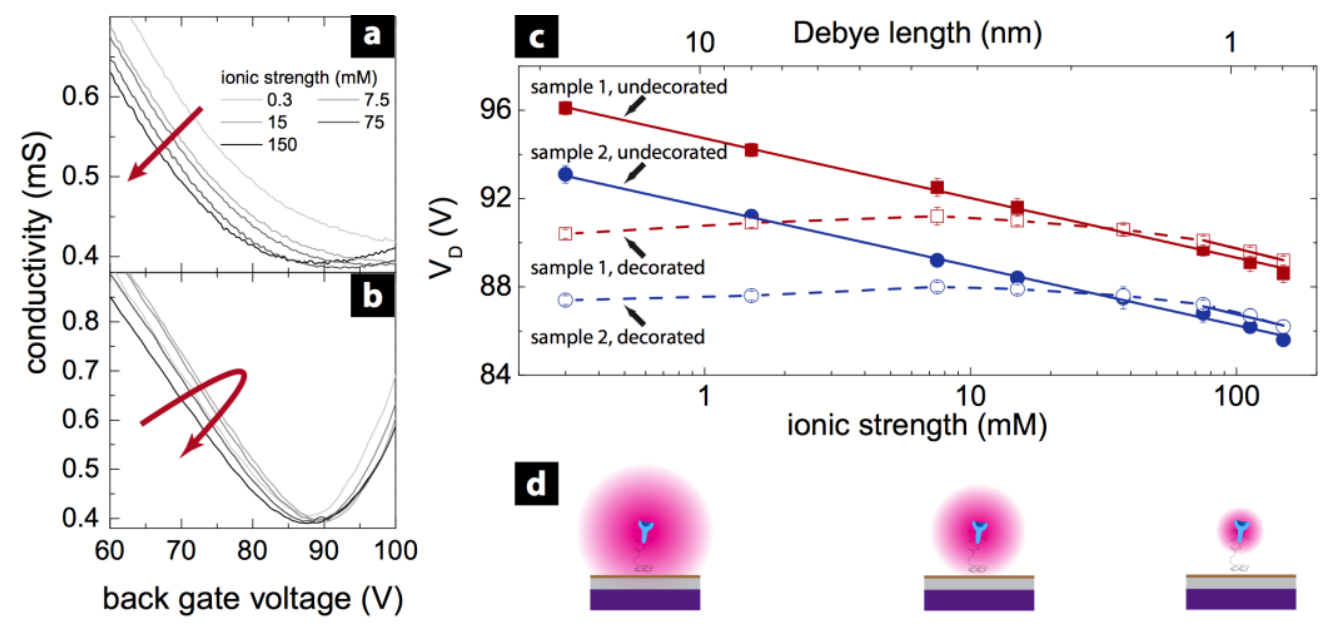

Figure 2. (a) Conductivity vs. back gate voltage for undecorated GFET sample 1 . The Dirac voltage decreases monotonically with ionic strength $c$. (b) Conductivity vs. back gate voltage for the same device decorated with wsMOR. The Dirac voltage behavior is non-monotonic. Arrows in (a) and (b) indicate the shift of Dirac voltage as $c$ is increased. (c) Dirac voltage vs. ionic strength for two devices (Sample 1 is featured in panels (a) -(b)). Solid lines are fits to the data with formalism described in the Section 4 of the Supporting Information. (d) Schematic illustration of ionic screening of the electrostatic field due to charges on wsMOR, at ionic strengths (Debye screening lengths) indicated on the bottom (top) $x$-axis of panel (c). 
We explored ionic screening effects for bare and wsMOR-decorated GFET devices through measurements of the Dirac voltage in solution with varying ionic strength (See Supporting information for Dirac voltage extraction). For undecorated GFETs, the inferred value of $V_{D}$ (solid points in Fig. 2a,b,c) decreases with ionic strength with a characteristic log-linear behavior that reflects increased ionic screening of the charges in the Stern layer ${ }^{9,10,14,16}$ (See Section 1 of the Supporting Information for additional details). Strikingly, for wsMOR-decorated GFETs the Dirac voltage exhibits a different, non-monotonic dependence on ionic strength (Fig. 2b,c). The Dirac voltage increases with ionic strength until a critical "knee" point at ionic strength $\sim 10 \mathrm{mM}\left({ }_{D} \sim\right.$ $3 \mathrm{~nm}$ ), after which the slope of the curve decreases and changes sign from +0.6 to -3.0 (Fig. 2c). Undecorated or decorated, samples 1 and 2 show identical behavior except for an offset in $V_{D}$ that is ascribed to different charge trap densities for the two substrates.

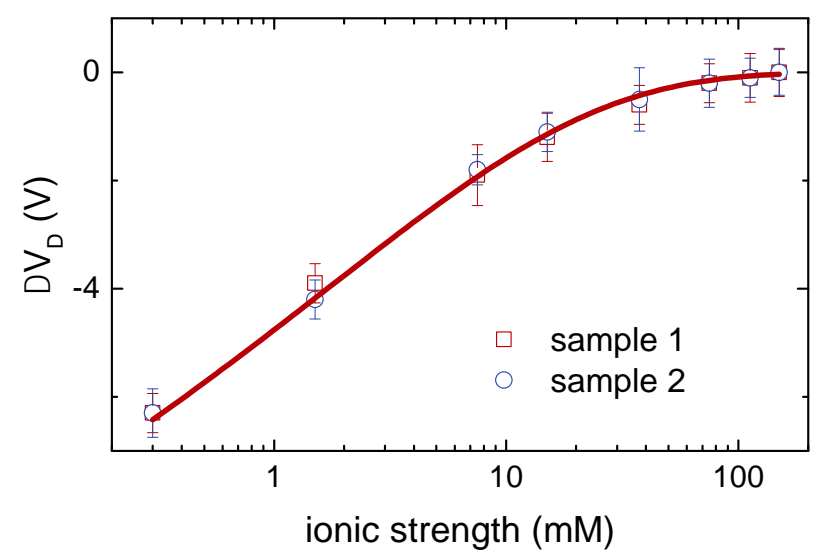

Figure 3. Difference between the Dirac voltages for two devices, before and after decoration with wsMOR. The saturation value of $\Delta V_{D}$ (see Fig. 2c) has been suppressed. The red solid curve is a fit to the data using Eqn. (1) from the main text.

Proposed mechanisms for these observations involve protonated residues on the wSMOR $(\sim 70$ total), which provide either (Faradaic) electron transfer ${ }^{20}$ or chemical gating due to protonation $^{32}$. At low ionic strength $(0.3 \mathrm{mM})$, the difference in Dirac voltage between 
undecorated and decorated versions of the same device, $\Delta V_{D} \sim-6 \mathrm{~V}$ (Fig. 2c and 3), corresponding to carrier density of $4000 \mathrm{e}^{-} \mu \mathrm{m}^{-2}$, or $\sim 35$ electrons per bound wsMOR. The Faradaic model requires a charge transfer efficiency of $\sim 55 \%$, which is implausibly large and greater by an order of magnitude than reports of $4 \%$ for primary amine groups near carbon nanotube devices ${ }^{12,38}$. In contrast, this carrier density is readily explained quantitatively by considering the combined effect of basic and acidic protein residues subject to protonation and deprotonation, respectively, in solution. To estimate the net charge of the wsMOR, we consider pKa values for the relevant amino acids in the free state (44 lysine, 15 arginine, 14 histidine, 8 aspartic acid , and 25 glutamic acid ) and find a net of $\sim+38$ fundamental charges per wsMOR, in good agreement with the data.

To our knowledge the problem of chemical-gating effect by charged biomolecules in ionic solution has not been solved analytically. We therefore analyze the problem approximately by assuming that the graphene acts as a perfect electrical conductor, and the protein molecule has a spherically symmetric charge distribution whose electric field decreases exponentially into the diffuse layer due to ionic screening on the scale of the Debye length. In this approximation, the Dirac voltage change $\Delta V_{D}$ induced by wsMOR decoration can be found with the image charge technique ${ }^{33,34}$ :

$$
V_{D}=\frac{4{ }_{0} A_{A}}{C_{o x}} \frac{2 k_{B} T}{e} \sinh ^{1} \frac{{ }_{M}}{\sqrt{8{ }_{0} k_{B} T c}} a e^{\frac{a}{D}},
$$

where ${ }_{A}$ is the wsMOR density of $115 \mu \mathrm{m}^{-2},{ }_{M}$ is the net surface charge density of the wsMOR, and $a$ is the distance from the graphene to the center of the wsMOR molecule. This result is analogous to the Debye-Hückel model for screening of a point charge by ionic solution ${ }^{6,35}$. We note that $\Delta V_{D}$ depends exponentially on the Debye length, in agreement with 
the observation that the value of $\Delta V_{D}$ tends towards zero for ionic strength $\sim 10 \mathrm{mM}$, where ${ }_{D} \quad a$ and the knee point of $V_{D}$ for the decorated GFETs as shown in Fig. 2c.

As shown in Fig. 3b, the data for $\Delta V_{D}$ as a function of ionic strength $I$ are very well described by Eqn. (1), where the fit parameters are the distance $a$ and the charge density ${ }_{M}$; the best fit parameter values for $a$ and ${ }_{M}$ are $3.5 \pm 0.5 \mathrm{~nm}$ and $0.11 \pm 0.02 \mathrm{C} \mathrm{m}^{-2}$ respectively. Based on the coupling chemistry used in the experiment, $a$ is expected to be $\sim 3.1 \mathrm{~nm}$, in good agreement with the fit. With the approximation that the radius of the ion-shell outside the wsMOR is approximately the radius of wsMOR plus the thickness of the Stern layer (a total of $\sim 2.3 \mathrm{~nm}$ ), the best fit value of the charge density ${ }_{M}$ corresponds to $46 \pm 9$ positive charges per wsMOR molecule, in good agreement with $\sim 38$ positive sites per wsMOR that was estimated above by considering charging of the wsMOR residues in solution.

\section{Conclusions}

We used fabricated clean and bio-compatible GFETs and decorated them with wsMOR using a bifunctional linker molecule. We measured the Dirac voltage shift as a function of ionic strength and provided a quantitative explanation based on accepted theory for the electric double layer and a simplified model of protein electrostatics. The results are fully consistent with the action of solution-mediated chemical-gating by bound wsMOR molecules as the mechanism that shifts the Dirac voltage of graphene in our biosensor system. This investigation shows the potential of this system for studying the charging properties of biomolecules bound to the FET channel based on graphene or other two-dimensional materials. 


\section{AUTHOR INFORMATION}

\section{Corresponding Author}

cjohnson@sas.upenn.edu

\section{Funding Sources}

This work was supported by the Defense Advanced Research Projects Agency (DARPA) and the U.

S. Army Research Office under grant number W911NF1010093. Additional support is

acknowledged from: National Science Foundation Accelerating Innovation in Research program

AIR ENG1312202, the Nano/Bio Interface Center NSF NSEC DMR0832802, FAER (Foundation for

Anesthesia Education and Research, PI, RL), NIH K08 (K08-GM-093115-01) (PI, RL), NIH R01

1RO1GM111421-01, and GROFF (PI, RL), and the Department of Anesthesiology and Critical Care at the University of Pennsylvania (PI, RL).

\section{ACKNOWLEDGMENT}

JGS acknowledges infrastructural support from the Penn LRSM MRSEC (NSF DMR1120901).

\section{Reference}

1 Bendali, A. et al. Purified Neurons can Survive on Peptide- Free Graphene Layers. Advanced Healthcare Materials (2013).

2 Chung, C. et al. Biomedical applications of graphene and graphene oxide. Acc. Chem. Res. 46, 2211-2224 (2013).

3 Gao, N. et al. General Strategy for Biodetecion in High Ionic Strength Solutions Using Transistor-Based Nanoelectronic Sensors. Nano Letters 15, 2143-2148 (2015).

4 Elnathan, R. et al. Biorecognition layer engineering: overcoming screening limitations of nanowire-based FET devices. Nano Lett. 12, 5245-5254 (2012).

5 Patolsky, F., Zheng, G. \& Lieber, C. M. Fabrication of silicon nanowire devices for ultrasenstive, label-free, real-time detection of biological and chemical species. Nature Protocols 1, 1711-1724 (2006).

6 Sorgenfrei, S., Chiu, C.-Y., Johnston, M., Nuckolls, C. \& Shepard, K. Debye screening in single-molecule carbon nanotube field-effect sensors. Nano Lett. 11, 3739-3743 (2011). 
7 Choi, Y. et al. Single-molecule Lysozyme dynamics monitored by an electronic circuit. Science 335, 319-324 (2012).

8 Choi, Y. et al. Dissecting single-molecule signal transduction in carbon nanotube circuits with protein engineering. Nano Letters 13, 625-631 (2013).

9 Heller, I. et al. Influence of electrolyte composition on liquid-gated carbon nanotube and graphene transistors. J. Am. Chem. Soc. 132, 17149-17156 (2010).

10 Ohno, Y., Maehashi, K., Yamashiro, Y. \& Matsumoto, K. Electrolyte-gated graphene field-effect transistors for detecting $\mathrm{pH}$ and protein adsorption. Nano Lett. 9, 3318-3322 (2009).

11 Yan, J., Zhang, Y., Kim, P. \& Pinczuk, A. Electric field effect tuning of electronphonon coupling in graphene. Phys. Rev. Lett. 98, 166802 (2007).

12 Israelachvili, J. Intermolecular and surface forces: Revised third edition. (Acadmeic Press, 2011).

13 Artyukhin, A. B. et al. Controlled Electrostatic Gating of Carbon Nanotube FET Devices. Nano Lett. 6, 2080-2085 (2006).

14 Chen, F., Qing, Q., Xia, J., Li, J. \& Tao, N. Electrochemical gate-controlled charge transport in graphene in ionic liquid and aqueous solution. J. Am. Chem. Soc. 131, 9908-9909 (2009).

15 Bonthuis, D., Gekle, S. \& Netz, R. Dielectric profile of interfacial water and its effect on double-layer capacitance. Phys. Rev. Lett. 107, 166102 (2011).

16 Hess, L. et al. Graphene transistor arrays for recording action potentials from electrogenic cells. Adv Mater 23, 5045-5049 (2011).

17 Uesugi, E., Goto, H., Eguchi, R., Fujiwara, A. \& Kubozono, Y. Electric doublelayer capacitance between an ionic liquid and few-layer graphene. Scientific Reports 3 (2013).

18 Lerner, M. et al. Toward Quantifying the Electrostatic Transduction Mechanism in Carbon Nanotube Molecular Sensors. J. Am. Chem. Soc. 134, 14318-14321 (2012).

19 Bradley, K., Briman, M., Star, A. \& Grüner, G. Charge transfer from adsorbed proteins. Nano Letters 4, 253-256 (2004).

20 Bradley, K., Gabriel, J.-C., Briman, M., Star, A. \& Grüner, G. Charge transfer from ammonia physisorbed on nanotubes. Phys. Rev. Lett. 91, 218301 (2003).

21 Farid, S. et al. Detection of interferon gamma using graphene and aptamer based FET-like electrochemical biosensor. Biosensors and Bioelectronics 71, 294-299 (2015).

22 Lerner, M. et al. Scalable Production of Highly-Sensitive Nanosensors Based on Graphene Functionalized with a Designed G Protein-Coupled Receptor. Nano Lett. (2014).

23 Butler, S. et al. Progress, challenges, and opportunities in two-dimensional materials beyond graphene. ACS Nano 7, 2898-2926 (2013).

24 Sarkar, D. et al. MoS2 Field-Effect Transistor for Next-Generation Label-Free Biosensors. ACS Nano 8, 3992-4003 (2014).

25 Perez-Aguilar, J. M. et al. A computationally designed water-soluble variant of a G-protein-coupled receptor: the human mu opioid receptor. PLoS One 8, e66009 (2013). 
26 Zhao, X. et al. Characterization of a computationally designed water-soluble human $\mu$-opioid receptor variant using available structural information. Anesthesiology 121, 866-875, doi:10.1097/ALN.0000000000000308 (2014).

27 Bayburt, T. H. \& Sligar, S. G. Membrane protein assembly into Nanodiscs. FEBS Lett. 584, 1721-1727 (2010).

28 Goldsmith, B. R. et al. Biomimetic Chemical Sensors Using Nanoelectronic Readout of Olfactory Receptor Proteins. ACS nano 5, 5408-5416, doi:10.1021/nn200489j (2011).

29 Lerner, M. B. et al. Scalable Production of Highly Sensitive Nanosensors Based on Graphene Functionalized with a Designed G Protein-Coupled Receptor. Nano Lett. 14, 2709-2714, doi:10.1021/n15006349 (2014).

30 Chen, R., Zhang, Y., Wang, D. \& Dai, H. Noncovalent sidewall functionalization of single-walled carbon nanotubes for protein immobilization. J. Am. Chem. Soc. 123, 3838-3839 (2001).

31 Kodali, V. et al. Nonperturbative chemical modification of graphene for protein micropatterning. Langmuir 27, 863-865 (2010).

32 Lerner, M. B. et al. Toward quantifying the electrostatic transduction mechanism in carbon nanotube molecular sensors. J. Am. Chem. Soc. 134, 14318-14321, doi:10.1021/ja306363v (2012).

33 Norde, W. \& Lyklema, J. Why proteins prefer interfaces. J. Biomater. Sci., Polym. Ed. 2, 183-202 (1991).

34 Purcell, E. M. \& Morin, D. J. in Electricity and Magnetism (Cambridge University Press, 2013).

35 Prisbrey, L., Schneider, G. \& Minot, E. Modeling the electrostatic signature of single enzyme activity. J. Phys. Chem. B 114, 3330-3333 (2010). 